\title{
Reconfigurable Quantum Local Area Network Over Deployed Fiber
}

\author{
Muneer Alshowkan $\odot,{ }^{1, *}$ Brian P. Williams $\odot,{ }^{1}$ Philip G. Evans $\odot,{ }^{1}$ Nageswara S.V. Rao $\odot,{ }^{2}$ \\ Emma M. Simmerman $\odot,{ }^{3}$ Hsuan-Hao Lu $\odot,{ }^{4}$ Navin B. Lingaraju $\odot,{ }^{4}$ Andrew M. Weiner $\odot,{ }^{4}$ \\ Claire E. Marvinney $\odot,{ }^{5}$ Yun-Yi Pai $\odot,{ }^{5}$ Benjamin J. Lawrie $\odot,{ }^{5}$ Nicholas A. Peters $\odot,{ }^{1}$ and \\ Joseph M. Lukens ${ }^{1, \dagger}$ \\ ${ }^{1}$ Quantum Information Science Group, Oak Ridge National Laboratory, Oak Ridge, Tennessee 37831, USA \\ ${ }^{2}$ Autonomous and Complex Systems Group, Oak Ridge National Laboratory, Oak Ridge, Tennessee 37831, USA \\ ${ }^{3}$ Department of Applied Physics, Stanford University, Stanford, California 94305, USA \\ ${ }^{4}$ School of Electrical and Computer Engineering and Purdue Quantum Science and Engineering Institute, Purdue \\ University, West Lafayette, Indiana 47907, USA \\ ${ }^{5}$ Materials Science and Technology Division, Oak Ridge National Laboratory, Oak Ridge, Tennessee 37831, USA
}

(Received 5 March 2021; accepted 30 August 2021; published 6 October 2021)

\begin{abstract}
Practical quantum networking architectures are crucial for scaling the connection of quantum resources. Yet quantum network testbeds have thus far underutilized the full capabilities of modern lightwave communications, such as flexible-grid bandwidth allocation. In this work, we implement flex-grid entanglement distribution in a deployed network for the first time, connecting nodes in three distinct campus buildings time synchronized via the Global Positioning System. We quantify the quality of the distributed polarization entanglement via log-negativity, which offers a generic metric of link performance in entangled bits per second. After demonstrating successful entanglement distribution for two allocations of our eight dynamically reconfigurable channels, we realize the first deployed fiber network demonstration of remote state preparation (RSP), a fundamental quantum communications protocol with utility for performing remote private "blind" quantum computing. We further demonstrate RSP not only at one location but over three nodes in three locations. In general, our results highlight an advanced paradigm for managing entanglement resources in quantum networks of ever-increasing complexity and service demands.
\end{abstract}

DOI: 10.1103/PRXQuantum.2.040304

\section{INTRODUCTION}

Quantum communications networks play a key role in advancing quantum information science (QIS). Some examples where quantum networks are crucial include distributed computing [1], enhanced sensing [2-4], secure communications [5,6], blind computing [7,8], and the highly anticipated quantum internet $[9,10]$. In the context of fundamental scientific discovery, quantum networks have potential to improve the sensitivity of astronomical interferometry $[11,12]$, and networks attaining entanglement-enhanced clock synchronization [13-15] should impact a variety of detection techniques based on

\footnotetext{
*alshowkanm@ornl.gov

†lukensjm@ornl.gov
}

Published by the American Physical Society under the terms of the Creative Commons Attribution 4.0 International license. Further distribution of this work must maintain attribution to the author(s) and the published article's title, journal citation, and DOI. distributed atomic clocks, including those being explored in dark matter searches [16,17].

Practical quantum key distribution (QKD) $[18,19]$ arguably the most mature quantum application - is designed for two-node links; in order to scale beyond two nodes for quantum digital signatures [20-22] or secret sharing [23-29], fully connected quantum network architectures are desirable.

Fundamentally, any architecture should support entanglement between distant parties on-demand as a core capability, ideally in an efficient and agile manner. Theoretical approaches to extend distance based on quantum repeaters [30] are promising; however, the current technology is still in very early stages. The current implementations of quantum networks at the logical level can be classified as point-to-point, trusted-node, point-to-multipoint, and fully connected. The simple point-to-point link is found in typical QKD implementations between two remote parties, traditionally known as Alice and Bob [18]. The trustednode network [31-39] consists of multiple point-to-point links in a partial mesh fashion, where optical links terminate in trusted nodes designed for a given Alice-Bob 
pair. Communications between distant nodes are enabled by intermediate nodes in a hop-by-hop paradigm [31,32], so that end-to-end security requires that all intermediary nodes be trusted. In the simplest case of point-tomultipoint networks, a passive beam splitter can be used to enable a node to communicate with one of any available nodes at random [40]. Alternatively, leveraging broadband frequency and polarization hyperentangled photons [41], dedicated entanglement can be established between any pair of users by assigning frequency-correlated wavelength channels. Fully connected quantum networks have recently been realized in this paradigm using nested dense wavelength division multiplexers (DWDMs) [42, 43]. Reconfigurable quantum links can be obtained by combining such DWDMs with transparent spatial switches [44-48], although the individual channel spacings and bandwidths remain fixed. By permitting flexible grid definitions in addition to spatial switching, a wavelengthselective switch (WSS) offers further improvements, and has recently been leveraged in fully connected quantum network designs utilizing adaptive resource provisioning [49,50].

Although showing great promise, results for fully connected quantum entanglement networks with dense wavelength allocation have been based on either loop-back fibers [43] or a tabletop configuration $[42,49,50]$ where all detection events occur in the same physical site. This simplification of time synchronization and data management procedures does not reflect the ultimate networking objective of nonlocal spatially distributed entanglement. Practically useful quantum networks must consist of spatially separated nodes with independent, heterogeneous quantum resources (stationary qubits, detectors, photon sources, etc.) synchronized to a common clock. The network architecture must be compatible with other networking topologies to enable forming larger networks. A quantum network will also need classical network capabilities in the form of a control plane for management and a parallel data plane for classical communications between the network nodes.

Here, we address these needs in the first dynamic, fully connected quantum local area network (QLAN) for entanglement distribution over deployed fiber. Combining both adaptive bandwidth provisioning and simultaneous distributed remote detection with off-the-shelf control systems, the three-building campus network has eight independent entanglement channels in the lowest-loss telecommunications transmission window that are dynamically and seamlessly remotely reconfigurable, thus allowing various configurations and bandwidth allocations without the need to add or remove any components in the setup. By measuring link throughput in terms of entangled bits per second (ebits/s), we validate the quality of each logical connection and show how different provisioning scenarios modify these "on demand" rates. Finally, to concretely demonstrate that the network supports quantum protocols, we realize remote state preparation (RSP) over all links, which to our knowledge is the first implementation of this quantum communications protocol in any deployed network. Overall, our layered network design and use of flex-grid lightwave technology furnish a promising concept and path toward more general quantum networks, including those supporting quantum computers and quantum sensors, ultimately establishing a framework on which a future quantum internet can be architected.

\section{SETUP}

\section{A. Network architecture}

The QLAN can be described in terms of functional layers shown in Fig. 1(a) in a manner analogous to the Transmission Control Protocol/Internet Protocol (TCP/IP) stack [51,52]. In general, this logical structure encompasses the physical medium, routing, protocols, and applications that describe the behavior of each node in the network. In this way, we seek to bring the quantum network architecture closer to practice using the layered model. In some ways, the quantum network layers may closely mirror their classical counterparts; e.g., the point-to-point links at the link layer are quite similar to optical channels provisioned over conventional network backbones using add-drop DWDM devices. On the other hand, quantum network architectures diverge from classical networks in crucial ways. For example, the no-cloning theorem prohibits making a perfect copy of an arbitrary unknown quantum state [53], thus preventing the use of conventional detection and retransmission techniques in optical routing. For this reason, quantum layers may not offer the same functionalities found in their classical counterparts (e.g., error detection and correction as in the TCP/IP stack link layer) [10], and may require fundamental refinements as the technology advances.

In the QLAN, the physical layer corresponds to the physical components where the encoded photons are transmitted, received, and manipulated over the communications medium (optical fiber in this case). Every user in the network is connected to the WSS via the deployed fiber [Fig. 1(b)]; thus, the physical connections of this network correspond to the star topology option depicted in Fig. 1(c). In the link layer, the WSS partitions the received bandwidth and dynamically routes the correlated spectral slices to particular output ports. In terms of entanglement distribution, the transport layer can be viewed as a logical fully connected mesh topology; each link forms a private point-to-point connection between two nodes as in the "Full Mesh" option in Fig. 1(c). Because of the nonlocal nature of quantum entanglement, private logical connections appear even without a corresponding direct physical link: in other words, a star physical topology - central source connected to all $N$ nodes - is able to 

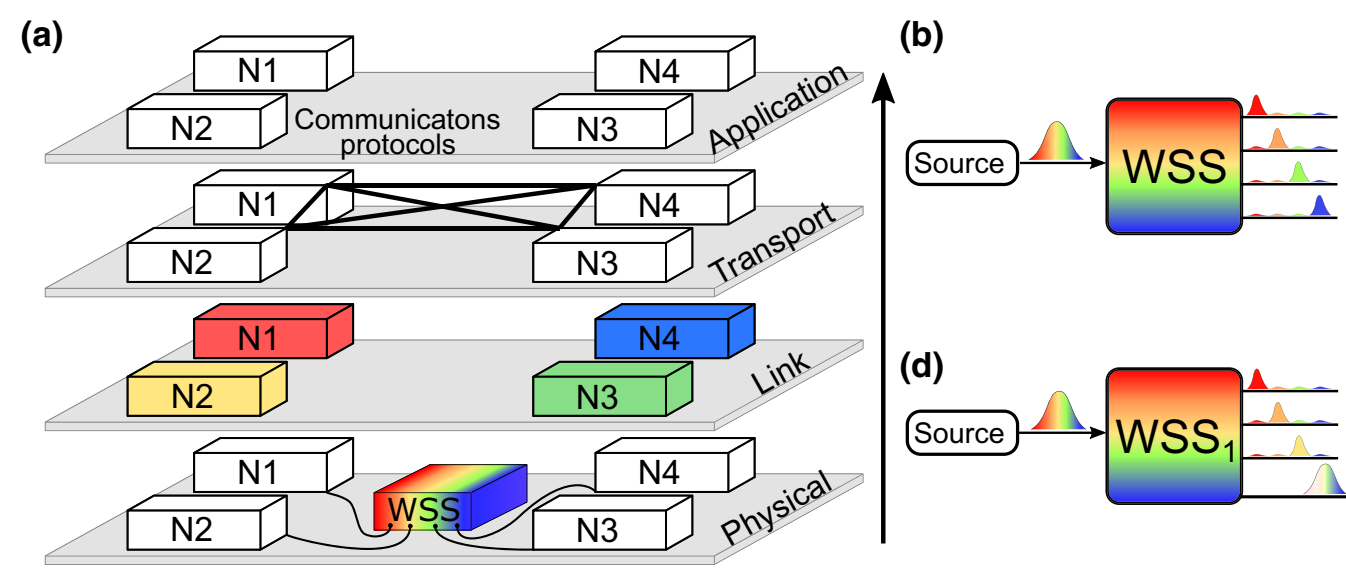

(c)

FIG. 1. (a) QLAN communication layers and services. The physical layer includes the optical components where the photons travel and are manipulated, the link layer slices the spectrum and routes each slice to a particular user, the transport layer is the quantumcorrelated network where a pair of users shares entanglement, and the application layer uses the entangled pairs to perform a service. (b) Single WSS configuration: the input spectrum is sliced and routed to the output ports. (c) Comparison of physical (star) and logical (fully connected mesh) topologies in network design. (d) Nested WSSs where a portion of the spectrum is routed from the first to the second WSS, thereby expanding the number of nodes connected by the entanglement source.

support a logical fully connected mesh, with entanglement connections between all $N(N-1) / 2$ user pairs. The application layer utilizes these logical connections to provide a quantum service between the connected users (e.g., QKD, teleportation, RSP). Building on similar ideas proposed in the design of quantum access networks [54,55], our architecture can be scaled by nesting multiple WSSs to create much larger networks as shown in Fig. 1(d). For example, the first WSS could send a section of the spectrum to the second WSS, creating a fully connected network where entangled links are formed between nodes in both WSSs. Note that in the QLAN stack here, we do not include a layer analogous to the internet layer found in the classical TCP/IP conceptual model [52]. Located between link and transport, the internet layer provides internetworking to connect multiple networks together - a capability not yet required in our single-network testbed.

At this early stage of development, any proposed quantum network stack is admittedly tentative, subject to refinements and modifications as quantum networks continue to mature. Yet by proposing such a stack here and identifying our QLAN's capabilities with relevant classical analogues, we are able to hint at the level of abstraction possible even with relatively simple quantum networks, in turn demonstrating a promising path forward for managing quantum resources in a scalable fashion.

\section{B. Source description}

Our QLAN utilizes an entangled photon source based on a 10-mm-long, periodically poled lithium niobate (PPLN) waveguide (HC Photonics), engineered for type-II spontaneous parametric down-conversion (SPDC). Pumping the PPLN waveguide with a continuous-wave laser $(\lambda=779.4$ $\mathrm{nm}$ for degenerate SPDC) followed by temporal walk-off compensation and spectral separation $[49,56]$, we can prepare signal-idler photon pairs that ideally are spectrally and polarization entangled, as described by the state

$$
\begin{aligned}
&|\psi\rangle \propto \int d \omega \Phi(\omega) {\left[\hat{a}_{H}^{\dagger}(\omega) \hat{a}_{V}^{\dagger}\left(2 \omega_{0}-\omega\right)\right.} \\
&\left.+e^{i \phi} \hat{a}_{V}^{\dagger}(\omega) \hat{a}_{H}^{\dagger}\left(2 \omega_{0}-\omega\right)\right]|\mathrm{vac}\rangle
\end{aligned}
$$

Here $\Phi(\omega)$ is the crystal phase-matching function with full-width at half-maximum (FWHM) bandwidth of approximately $2.5 \mathrm{~nm}(310 \mathrm{GHz}), \hat{a}_{H(V)}^{\dagger}(\omega)$ generates a photon at frequency $\omega$ with horizontal (vertical) polarization, and $\omega_{0}$ is half the pump frequency $\left(\omega_{0} / 2 \pi=\right.$ $192.3125 \mathrm{THz}$ ). The residual phase $\phi$ is compensated by the method described in Sec. III A, leaving the ideal Bell state $\left|\Psi^{+}\right\rangle=(1 / \sqrt{2})(|H V\rangle+|V H\rangle)$ for every pair of energy-matched frequencies. Further details regarding the preparation of the photon source can be found in Ref. [49].

This state is then sectioned into bands that are distributed to network users. We utilize a WSS to define eight pairs of frequency-correlated channels. Each channel $n$ has a width of $\Delta \omega / 2 \pi=25 \mathrm{GHz}$ and aligns to the International Telecommunication Union (ITU) grid (ITUT Rec. G.694.1) as listed in Table I, centered at $\omega_{n}=$ $\omega_{0} \pm \Delta \omega\left(n-\frac{1}{2}\right)$ for the signal (idler). These channels occupy an approximately 3-nm bandwidth in the C-band $(1557.3-1560.5 \mathrm{~nm})$.

We first characterize the spectral correlations and polarization entanglement of the source with two colocated receivers. For joint spectral intensity (JSI) characterization, we program the WSS to route one signal channel and one idler channel to two superconducting nanowire 
TABLE I. DWDM ITU 25-GHz grid C-band channel numbers of the signal and the idler photons. The pair of channels in the same row are entangled. Fidelities are with respect to the ideal polarization Bell state $\left|\Psi^{+}\right\rangle$, and both photons are measured locally at the source prior to transmission.

\begin{tabular}{|c|c|c|c|c|c|}
\hline \multirow[b]{2}{*}{ Ch. } & \multicolumn{2}{|c|}{ Signal } & \multicolumn{2}{|c|}{ Idler } & \multirow[b]{2}{*}{ Fidelity } \\
\hline & ITU & $\mathrm{THz}$ & ITU & $\mathrm{THz}$ & \\
\hline 1 & 23.25 & 192.325 & 23.00 & 192.300 & $0.952 \pm 0.007$ \\
\hline 2 & 23.50 & 192.350 & 22.75 & 192.275 & $0.948 \pm 0.007$ \\
\hline 3 & 23.75 & 192.375 & 22.50 & 192.250 & $0.942 \pm 0.008$ \\
\hline 4 & 24.00 & 192.400 & 22.25 & 192.225 & $0.935 \pm 0.007$ \\
\hline 5 & 24.25 & 192.425 & 22.00 & 192.200 & $0.944 \pm 0.006$ \\
\hline 6 & 24.50 & 192.450 & 21.75 & 192.175 & $0.949 \pm 0.006$ \\
\hline 7 & 24.75 & 192.475 & 21.50 & 192.150 & $0.943 \pm 0.007$ \\
\hline 8 & 25.00 & 192.500 & 21.25 & 192.125 & $0.947 \pm 0.007$ \\
\hline
\end{tabular}

single-photon detectors (SNSPDs) for coincidence measurement. Figure 2 shows the obtained JSI with a 5-ns coincidence window and 5-s integration time per measurement (at a pump power of approximately $17 \mathrm{~mW}$ ). The channel numbers for the 25-GHz-wide signal and idler bins correspond to the ITU mapping in Table I. Strong correlations are observed for energy-matched channels, with small crosstalk in the lower off-diagonal. The reduction in flux with increasing channel number matches the $310-\mathrm{GHz}$ FWHM of the type-II generation process, which places the $50 \%$ level inside Ch. 7. Overall, the coincidenceto-accidental ratio (average of matched bins divided by average of mismatched bins) is 11.4.

To characterize the quality of polarization entanglement for these eight channel pairs, we measure twophoton correlations in the $H / V$ and $D / A$ bases, defining $|D\rangle=(1 / \sqrt{2})(|H\rangle+|V\rangle)$ and $|A\rangle=(1 / \sqrt{2})(|H\rangle-|V\rangle)$. We employ the Bayesian tomography method of Ref. [57]

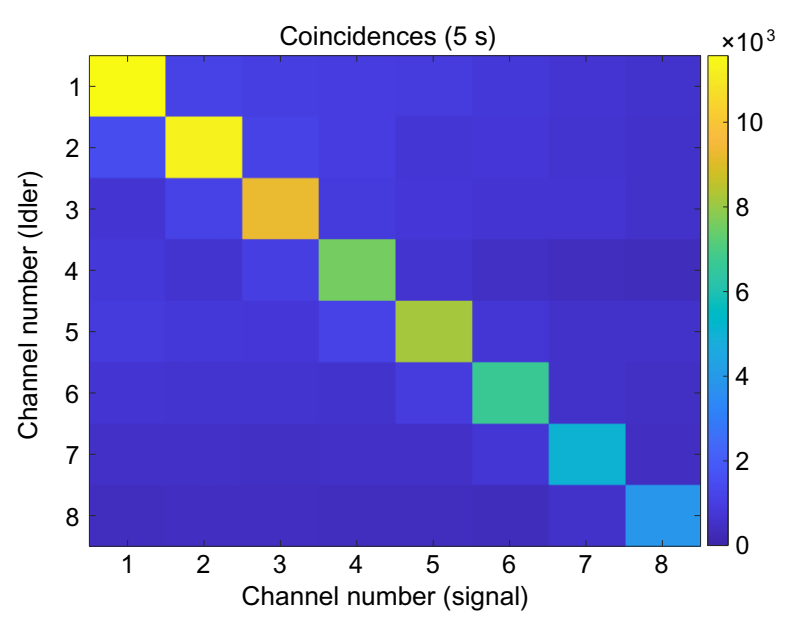

FIG. 2. Joint spectral intensity of the source measured locally with SNSPDs and plotted as a function of the signal and idler channel numbers. Each point is obtained with a 5-ns coincidence window and 5-s integration time. for quantum state reconstruction such that, though two sets of measurements (both in $H / V$ and $D / A$ bases) are not tomographically complete, we can still obtain meaningful state estimates with low error bars due to the highly correlated nature of our input state. We confirm high fidelity with respect to $\left|\Psi^{+}\right\rangle$for each individual biphoton channel as shown in Table I.

\section{Time synchronization}

As every node in the network is spatially distributed, independent receivers are required to evaluate network performance and perform quantum communications tasks. For accurate photon coincidence counting, all time-todigital converters (TDCs) in the network must be synchronized to a common clock. Such distributed timing synchronization presents a critical technical challenge in extending tabletop quantum networking experiments to the field. And the timing requirements in quantum communications are frequently much tighter than for classical LANs. For example, the precision time protocol (PTP) is a simple and ubiquitous standard for synchronizing devices over ethernet, yet it is designed to attain only submicrosecond jitter [58], whereas subnanosecond precision is desired in many photon counting quantum networking experiments. As a major improvement on PTP, White Rabbit [59] leverages synchronous ethernet to attain picosecondlevel precision. Accordingly, White Rabbit appears particularly promising for future quantum networks, but requires protocol-compatible ethernet infrastructure. Alternatively, as lower-level, fully optical approaches, quantum communications experiments have utilized optical sync pulses or direct tracking of either pulsed quantum signals $[46,60]$ or photon coincidence peaks [61-64] to compensate for the drift in independent clocks.

In our QLAN, we select GPS-based synchronization for its simplicity, cost-effectiveness, and availability (a GPS antenna was already available in one lab). From the GPS signal, both time pulses - at a rate of one pulse per second (PPS) - and a $10 \mathrm{MHz}$ clock can be derived. Before deployment on our network, we characterize the jitter between time pulses produced by pairs of independent receivers (Trimble Thunderbolt E). Histograms of the relative delays recorded over several hours using a time interval counter (Stanford Research Systems SR620) appear in Fig. 3, for two pairs of the three devices. The distributions of relative delays have standard deviations of $12.1 \mathrm{~ns}$ for the devices of Alice and Bob [Fig. 3(a)] and $14.7 \mathrm{~ns}$ for Charlie and Alice [Fig. 3(b)]. This suggests that coincidence windows up to approximately $30 \mathrm{~ns}$ would be reasonable in our network; in practice, we find a window of 10 ns to offer a reasonable balance between counting photon pairs and reducing the noise from accidentals. At these jitters, GPS offers significantly higher precision than PTP, though upgrading to a system reaching 
(a)

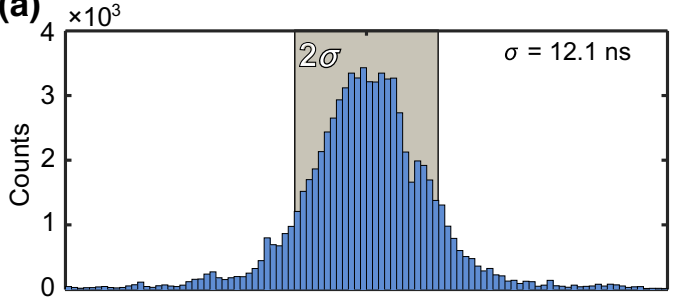

(b)

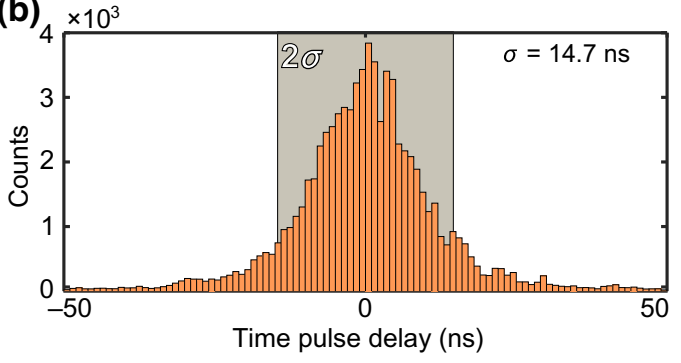

FIG. 3. Histogram of relative delays between time pulses from two GPS receivers. (a) Alice and Bob. (b) Charlie and Alice.

subnanosecond levels would be an important improvement moving forward.

\section{IMPLEMENTATION}

\section{A. Deployed network}

The experiment is performed in three buildings on the Oak Ridge National Laboratory (ORNL) campus, as depicted in Fig. 4. The source and Alice are colocated in the same lab while Bob and Charlie reside in separate buildings, connected to the source through total fiber path lengths of approximately 250 and $1200 \mathrm{~m}$, respectively. The source of the polarization-entangled photons and each user in the network are connected to a WSS by a single fiber, where the outputs pass through a manual fiber-based polarization controller (FPC) to compensate for the polarization rotation along the fiber path. The output of the FPC goes directly to the polarization analyzer for Alice and to the fiber patch panel for Bob and Charlie. From the patch panel, the fibers traverse multiple communication rooms before reaching the panels at Bob and Charlie. The fiber from the patch panel in each location is then routed to the polarization analyzer. The output of Bob's polarization analyzer is sent directly to an InGaAs avalanche photodiode (APD), whereas for Alice and Charlie, the outputs are directed to SNSPDs preceded by FPCs to maximize detection efficiency.

While each user could employ a local antenna, it is simpler in our case to share the GPS signal from the antenna at Bob's location with the other nodes via rf over fiber (RFOF). The rf GPS signal at Bob is connected to a commercial RFOF transmitter that outputs an optical signal. This is subsequently split and sent over separate strands of the deployed fiber to every node's RFOF receiver, which then outputs the original rf GPS signal. The GPS receivers at each network node synchronize a FPGA-based TDC with the $10-\mathrm{MHz}$ and $1-\mathrm{Hz}$ (PPS) clocks; we bin each detection event to 5-ns resolution using the FPGA's internal $200-\mathrm{MHz}$ clock, tracking the number of $10-\mathrm{MHz}$ and

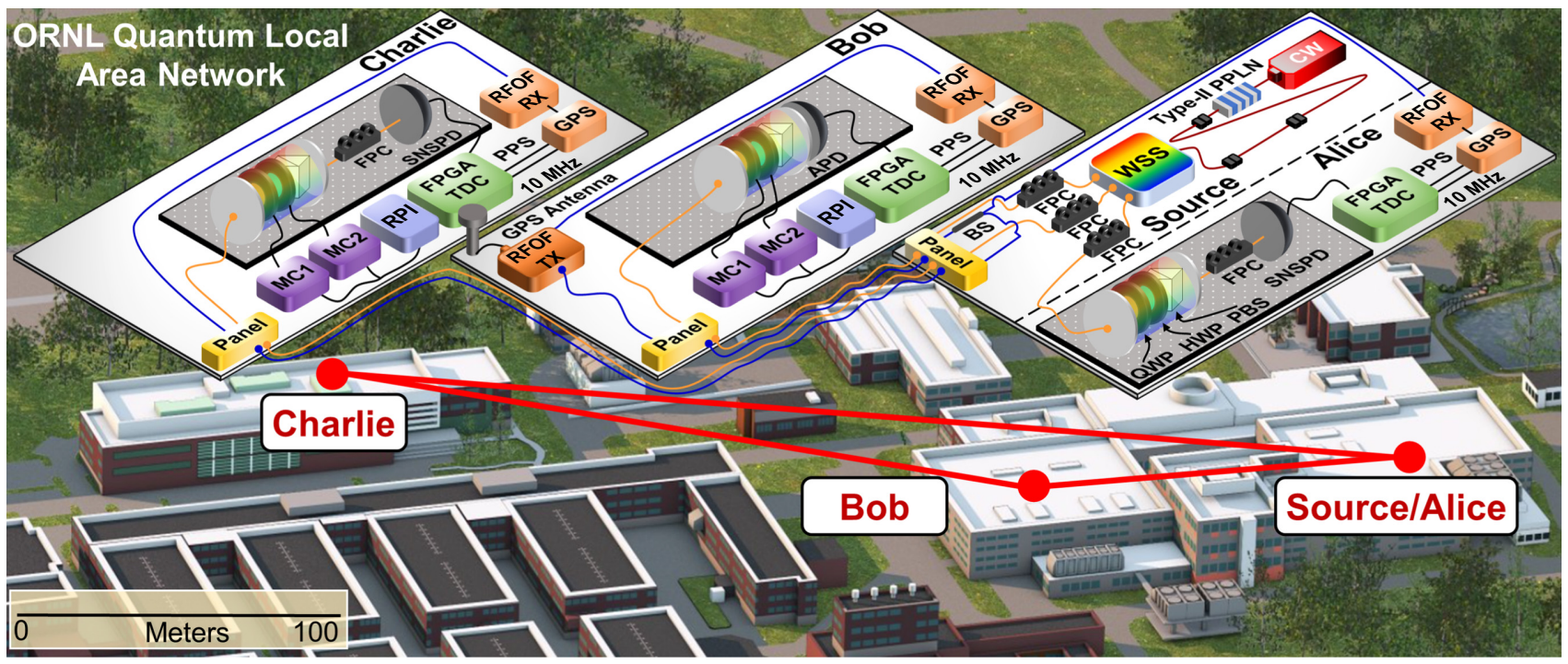

FIG. 4. Map of QLAN on the ORNL campus. The receiver configurations at each node are shown as insets. APD, avalanche photodiode; CW, continuous-wave laser; FPC, fiber polarization controller; FPGA, field-programmable gate array; GPS, Global Positioning System; HWP, half-wave plate; MC, motion controller; Panel, fiber-optic patch panel; BS, beam splitter; PBS, polarizing beam splitter; PPLN, periodically poled lithium niobate; PPS, pulse per second; Source, entanglement source; QWP, quarter-wave plate; RFOF RX, rf over fiber receiver; RFOF TX, rf over fiber transmitter; RPI, Raspberry Pi microprocessor board (to control MCs); SNSPD, superconducting nanowire single-photon detector; WSS, wavelength-selective switch. 
1-Hz cycles to assign a global network timestamp. Note that our approach offers a general and scalable framework for increasing the network size, as bringing an additional node online requires a single-photon detector and relatively inexpensive off-the-shelf electronic components.

For data transfer and instrument control between the nodes, we set up a classical network managed by a control plane for routing over the campus networking infrastructure. Events recorded by each TDC are shared over the control plane for data analysis and coincidence counting. This dedicated network allows timely and accurate data transfer for analysis and monitoring. It is crucial to have a classical network with bandwidth commensurate with the quantum data gathered. In our experiments, each time stamp is a 32bit record, and events occur at rates up to approximately $10^{5} \mathrm{~s}^{-1}$ at the SNSPD nodes, corresponding to an average data rate of approximately $3 \mathrm{Mb} / \mathrm{s}$ - comfortably below the approximate $1 \mathrm{~Gb} / \mathrm{s}$ supported by our control plane.

With quantum information encoded in polarization in the QLAN, each node holds a polarization analyzer, a single-photon detector, and a TDC. Components of the analyzer include a quarter-wave plate (QWP), half-wave plate (HWP), and polarizing beam splitter (PBS). The angles of the waveplates at Bob's and Charlie's locations are set remotely with motion controllers, and manually at Alice's location. Alice and Charlie possess SNSPDs with efficiency $\eta \geq 80 \%$, whereas Bob uses an APD with efficiency $\eta \approx 20 \%$, dead time $100 \mu \mathrm{s}$, and gate window 33.5 ns at $15 \mathrm{MHz}$. The heterogeneous detectors result in widely varying link efficiencies and reflect the types of variability in quantum resources that should be expected in larger quantum networks.

Because of random birefringence effects induced by the single-mode optical fiber, careful compensation of polarization rotation must be performed in order to realize high-fidelity entanglement distribution. Given the fact that all fibers are located either indoors or underground, we do not find it necessary to perform active polarization tracking $[65,66]$; the polarization state is typically stable for hours at a time. However, we do perform manual compensation before each experiment, utilizing FPCs at the outputs of the WSS. Following an alignment procedure similar to previous fiber-based polarization sources [67], we first insert a polarizer after the biphoton source that permits only $H$ polarized photons to pass through, adjusting each FPC to minimize the counts when the analyzers are set to measure the $V$ state, i.e., fast axis of the QWP (HWP) oriented at $0^{\circ}$ $\left(45^{\circ}\right)$ with respect to horizontal. This ensures compensation of $H / V$ individually, up to an unknown phase between $H$ and $V$.

This residual phase amounts to an output state of the form $|H V\rangle+e^{i \phi}|V H\rangle$, which we can compensate by defining effective $D / A$ axes. That is, setting the fast axis of the QWP for both receivers to $45^{\circ}$ with respect to the horizontal, we tune the HWP angle on one of the two photons to maximize coincidences and define the $D / D$ measurement for both receivers: all other basis state projections are obtained by rotating the HWP by fixed amounts relative to each $D$ setting. This correction can be understood intuitively on the Poincaré sphere as a rotation that maintains an equal $H / V$ superposition but changes the relative phase between them [68]. Specifically, defining a QWP and HWP angle pair $\left(\theta_{Q}, \theta_{H}\right)$, we have the measurement settings $H=\left(0^{\circ}, 0^{\circ}\right), V=\left(0^{\circ}, 45^{\circ}\right), D=\left(45^{\circ}, x^{\circ}\right), A=$ $\left(45^{\circ}, x^{\circ}+45^{\circ}\right), R=\left(45^{\circ}, x^{\circ}+22.5^{\circ}\right)$, and $L=\left(45^{\circ}, x^{\circ}-\right.$ $22.5^{\circ}$ ), where $x$ is a free parameter chosen to maximize contrast. In the current experiment, we optimize the $x$ of one polarization analyzer individually for each pair of nodes, but it is important to note that the number of free parameters (three) is sufficient to optimize the HWP settings for all pairs of nodes simultaneously, by solving a system of equations or monitoring correlations in complementary bases in real time, similar to the techniques mentioned in Refs. $[42,69]$.

\section{B. Bandwidth Allocation 1}

The eight-channel polarization-entangled source supports a variety of bandwidth allocations that can be tailored to match a desired network configuration. Through the WSS, adjustments to bandwidth provisioning can be made in real time without modifying any fiber connections. This approach for entanglement distribution was recently realized in a tabletop experiment [49], and here we demonstrate it in a deployed fiber-optical network for the first time.

Given the imbalance in system efficiency, due to both the deployed fiber loss and heterogeneous detector technology, we explore and test two different bandwidth allocations. A patch-panel to patch-panel link loss of $1.8 \mathrm{~dB}$ ( $3.3 \mathrm{~dB})$ is measured from Alice to Bob (Charlie); combined with the detectors used, Charlie and Alice enjoy the highest overall efficiency, followed by Alice and Bob and finally Bob and Charlie. In the first allocation, we seek to balance the entanglement rates by assigning the spectral slice with lowest flux (Ch. 8) to Charlie and Alice (C-A), the brightest slice (Ch. 1) to Alice and Bob (A-B), and the remaining (Chs. 2-7) to Bob and Charlie (B-C).

At a pump power of $15.6 \mathrm{~mW}$, the average single counts in this allocation for each pair (in units of $\mathrm{s}^{-1}$ ) are: $1.2 \times$ $10^{5}$ and $5.0 \times 10^{3}(\mathrm{~A}-\mathrm{B}), 7.5 \times 10^{3}$ and $1.4 \times 10^{5}(\mathrm{~B}-\mathrm{C})$, and $1.0 \times 10^{4}$ and $4.7 \times 10^{4}(\mathrm{C}-\mathrm{A})$. To measure state quality, we perform polarization tomography on data obtained with a $10-n s$ coincidence window and 60-s integration time. Figure 5(a) shows the Bayesian mean estimated [57] density matrices for each pair of nodes, again based on measurements in the $H / V$ and $D / A$ bases. In these tests, only the logical link under test is bright (the other channels are blocked) to reduce the background at each detector; this is equivalent to spectrally resolved detection at each 
(a)
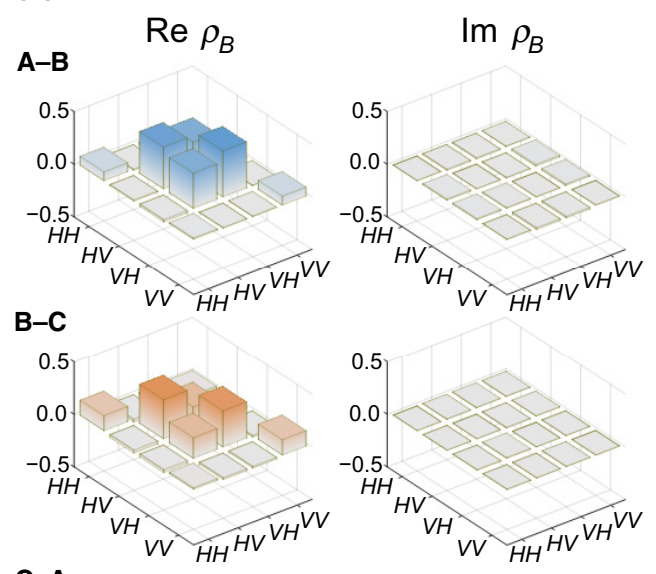

C-A
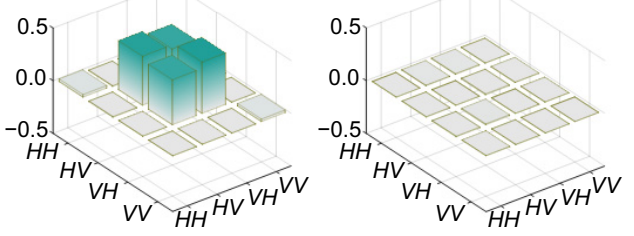

(b)
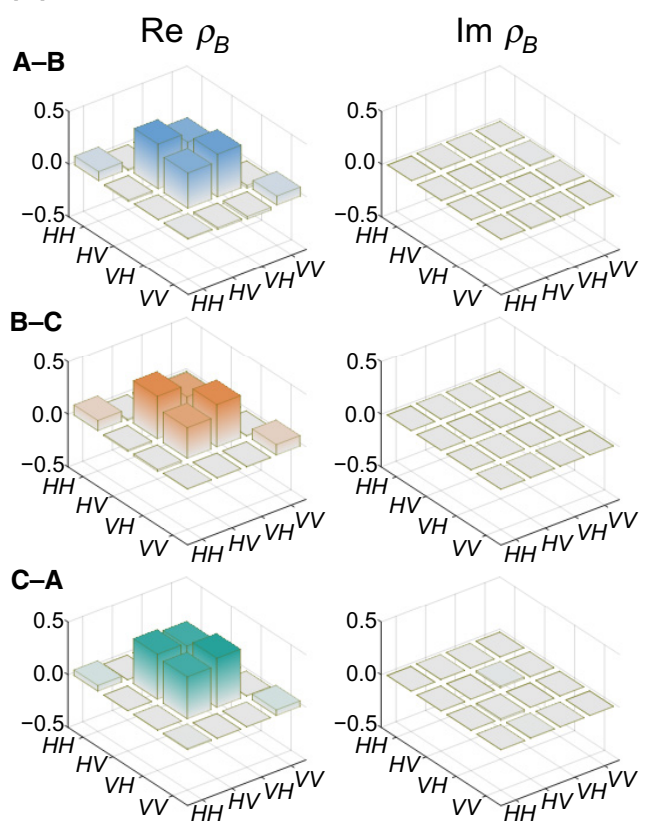

FIG. 5. Density matrices estimated by polarization tomography for each pair of users for (a) Allocation 1 and (b) Allocation 2. node. Nevertheless, no accidentals are subtracted from these results, and the fidelities with respect to $\left|\Psi^{+}\right\rangle$follow in Table II.

From fidelities alone, it is not immediately clear how useful the provided entanglement in the network is for generic quantum communications tasks. Since we have performed full state tomography, this could in principle be predicted for any desired protocol. But for greater generality, we propose an "entanglement bandwidth" analogous to bit rates in classical links. Distillable entanglement- the asymptotic rate of pure Bell pairs that could be produced from copies of the state, local operations, and classical communications [70] - seems especially fitting as such a quantifier, but it is extremely difficult to compute, even with full knowledge of the density matrix. As an alternative, we consider the log-negativity $E_{\mathcal{N}}$ [71], which provides an upper bound on distillable entanglement. (For two qubits specifically, $E_{\mathcal{N}}>0$ is also a necessary and sufficient condition for nonseparability [72].) When multiplied by the coincidence rate, $E_{\mathcal{N}}$ thus gives a generic metric for link performance in terms of entangled bits (ebits) per second $\left(R_{E}\right)$. Computing $E_{\mathcal{N}}$ and $R_{E}$ from the Bayesian samples, we obtain the results in the final two columns of Table II. Importantly, even the lowest-fidelity link (B-C) possesses an $R_{E}$ greater than zero by approximately three standard deviations.

\section{Bandwidth Allocation 2}

In the second allocation, our goal is to improve the lowest fidelity in the network and test the performance of different allocations for the other links. In general, a trade-off exists when selecting channel allocations: assigning more channels to a pair of users increases the number of entangled photons, boosting the coincidence rate, yet it can also result in lower fidelity. In the regime where noise is primarily due to accidentals from multipair emission, increasing the number of channels lowers the signal-to-noise ratio, since desired coincidences scale linearly and accidentals quadratically with flux [73]; similarly, broader bandwidth implies higher sensitivity to wavelength-dependent birefringence effects. When the wavelength-dependent phase shift in birefringent fiber is averaged over a larger bandwidth, the coherence of the polarization state is reduced

TABLE II. Link data for both bandwidth allocations.

\begin{tabular}{lccccc}
\hline \hline Allocation & Link & Ch. & Fidelity & $E_{\mathcal{N}}$ (ebits) & $R_{E}$ (ebits/s) \\
\hline 1 & A-B & 1 & $0.75 \pm 0.03$ & $0.70 \pm 0.08$ & $56 \pm 6$ \\
& B-C & $2-7$ & $0.55 \pm 0.06$ & $0.4 \pm 0.2$ & $30 \pm 10$ \\
& C-A & 8 & $0.90 \pm 0.01$ & $0.89 \pm 0.03$ & $206 \pm 6$ \\
2 & A-B & 3 & $0.75 \pm 0.03$ & $0.70 \pm 0.09$ & $57 \pm 7$ \\
& B-C & $1-2$ & $0.69 \pm 0.04$ & $0.6 \pm 0.1$ & $26 \pm 4$ \\
& C-A & 4 & $0.84 \pm 0.02$ & $0.82 \pm 0.05$ & $320 \pm 20$ \\
\hline \hline
\end{tabular}


as in Refs. $[68,74,75]$. How these effects play out in the overall entanglement rate $R_{E}$ is difficult to predict. So to explore the interplay between them, we next assign Chs. $1-2$ to B-C, Ch. 3 to A-B, Ch. 4 to $\mathrm{C}-\mathrm{A}$, leaving the remaining four channels unassigned as examples of resources available for future use should additional nodes come online.

The average single counts in Allocation 2 (units of $\mathrm{s}^{-1}$ ) are: $1.2 \times 10^{5}$ and $5.1 \times 10^{3}(\mathrm{~A}-\mathrm{B}), 6.4 \times 10^{3}$ and $6.7 \times 10^{4}(\mathrm{~B}-\mathrm{C})$, and $1.9 \times 10^{4}$ and $8.4 \times 10^{4}(\mathrm{C}-\mathrm{A})$. The density matrices obtained follow in Fig. 5(b), along with the specific fidelities and entanglement rates in Table II. Compared with Allocation 1, the A-B link maintains similar performance in all categories. B-C experiences an appreciable increase in fidelity but, due to the reduced flux, a slightly lower $R_{E}$. Interestingly, the higher flux on the $\mathrm{C}-\mathrm{A}$ link reveals the exact opposite behavior: lower fidelity but higher $R_{E}$. These examples highlight that the optimal allocation may depend on a given objective: what characteristics are most valuable to the users on a network? In light of the variety of possible answers to this question, bandwidth provisioning with the WSS offers exceptional flexibility compared to a fixed wavelength configuration: it can adapt and meet changing demands, apportioning resources for seamless service to each link without physical disconnections [49].

\section{REMOTE STATE PREPARATION}

As a proof-of-principle application of our QLAN, we perform the RSP protocol between network nodes. RSP is a quantum communications protocol similar but simpler than teleportation. Like teleportation, it requires users to share entanglement and classical communications. However, RSP uses a single-photon measurement on half the entangled pair instead of Bell state analysis on the to-beteleported input photon and half the entangled pair. In RSP, the sender measures one photon of an entangled pair in order to prepare the remaining particle at the receiver in some desired quantum state [76-79]. We note that this is nondeterministic, though in some cases, the classical communications enable one to apply a unitary operation to yield the desired state, or to simply postselect the successful events as we do here. One can envision applications of RSP in many of the networking scenarios typically considered for quantum teleportation. For example, assuming the availability of quantum memories, Bell states can be distributed and stored at any time, say, during periods of low network utilization. Later, this preshared entanglement can be leveraged to transmit an arbitrary quantum state between users without additional use of the quantum channel, which may be difficult to access during peak hours. As another example, RSP can be used to generate the inputs in blind quantum computing protocols, whereby computations can be performed remotely and securely on an untrusted server [7,80]. In general, whenever the sender knows the state to be transmitted, RSP is able to offer the same functionality as teleportation while reducing both the quantum measurement complexity and the classical communications cost [78].

Within the network layer architecture of Sec. II A, RSP resides in the application layer, leveraging the logical connections provided by the transport layer-i.e., the entanglement resources as summarized in Table II. The ideal Bell state $\left|\Psi^{+}\right\rangle$is maximally correlated in all three standard polarization bases (rectilinear, diagonal, and circular): $\left|\Psi^{+}\right\rangle \propto|H V\rangle+|V H\rangle=|D D\rangle-|A A\rangle=|R R\rangle-$ $|L L\rangle$. (We adopt the conventions $|R\rangle \propto|H\rangle+i|V\rangle$ and $|L\rangle \propto|H\rangle-i|V\rangle$.) Thus, a simple RSP experiment follows from measuring in one of these bases and performing tomography on the remaining photon, comparing the result to the ideal case.

We implement RSP of a single state on each link of the network, utilizing Allocation 2 above. For link A-B, Alice prepares the state $|R\rangle$ at Bob by projecting her photon onto $|R\rangle$; for $\mathrm{B}-\mathrm{C}$, Charlie prepares the state $|V\rangle$ at Bob by projecting his photon onto $|H\rangle$; and for $\mathrm{C}-\mathrm{A}$, Alice prepares the state $|D\rangle$ at Charlie by projecting her photon onto $|D\rangle$. The results of Bayesian tomography on the prepared qubits are plotted on the Poincare sphere in Fig. 6, based on measurements in a complete set of two-dimensional mutually unbiased bases $(H / V, D / A$, and $R / L)$. Each cloud consists of 1024 samples from the posterior distribution, giving a visual indication of the uncertainty in each result [81]. Fidelities with respect to the target state are provided for each link, including error bars from the standard deviation of the retrieved samples. As expected, the $\mathrm{C}-\mathrm{A}$ link gives the highest fidelity and lowest uncertainty, followed by A-B and B-C. The experimentally measured RSP states are in excellent agreement with predictions based on the density matrices in Fig. 5(b): their fidelities with respect to the relevant partially traced density matrix are approximately 0.99 in all cases, confirming that the nonidealities in Fig. 6 result from the underlying entanglement quality and not errors in the RSP protocol. To our knowledge,

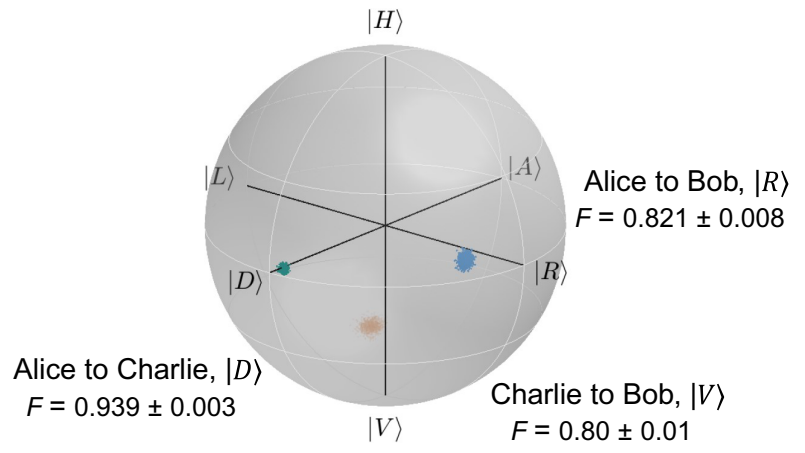

FIG. 6. Tomography results for qubit states remotely prepared on each logical link. 
these results represent the first demonstration of RSP over a deployed network, confirming the ability of our QLAN to provide the network stack needed to successfully carry out a quantum communications protocol.

\section{DISCUSSION}

In this work, we have successfully demonstrated the first adaptive bandwidth provisioning for entanglement in a deployed network, showing how allocations impact both fidelity and entanglement rates and enable dynamic quality-of-service provisioning. All results above were presented without accidental subtraction, confirming that appreciable entanglement is distributed to all user pairs under current conditions. However, from a technical perspective, reducing noise from accidentals would offer major performance improvements. Table III displays the results obtained for both allocations if we do subtract accidentals prior to tomography, where the accidentals correspond to the coincidences in a $10 \mathrm{~ns}$ window that is time shifted from the correlated peak. The fidelities in all cases are $>92 \%$, suggesting that addressing such noise limitations should improve the quality of service significantly. In comparison, the accidentals-subtracted fidelities of the individual channels before transmitting over the deployed fiber are 96\%-99\%, which suggests that, e.g., the B-C link of Allocation 1 is likely limited by imperfect birefringence compensation over the relatively large band allocated; detailed examination of each fiber's polarization transformation will therefore prove valuable in future studies. Intriguingly, the accidentals-subtracted entanglement rates $R_{E}$ in Table III are equal within error to the raw results in Table II. While we are unaware of any theoretical requirement for this relationship, intuitively it makes sense: subtracting accidentals eliminates noise from the data, but it does not increase the throughput of entangled pairs.

Perhaps the most effective technical direction to reduce noise would be improving the time synchronization across the QLAN. With the current GPS clocks, our coincidence windows are $10 \mathrm{~ns}$, yet the detector-jitter-limited coincidence peaks are much less than 1-ns wide. Accordingly, reducing network jitter - through an optical sync or White Rabbit - and using a 1-ns coincidence window would reduce accidentals tenfold while maintaining the number of correlated detections at the present level. Under such conditions, the added noise from all channels transmitting simultaneously would prove much more manageable. While our use of RFOF technology for GPS signal distribution is motivated by accessibility and cost, its use provides a clear upgrade path; since White Rabbit can provide the same timing signals as GPS $(1-\mathrm{Hz}$ and $10-\mathrm{MHz}$ clocks), White Rabbit switches and nodes can directly replace the RFOF transmitters and receivers in Fig. 4 with no additional changes to the network infrastructure.

In addition to cost, the choice between GPS and White Rabbit will depend in large part on the available transmission medium, with GPS appearing an excellent option for free-space channels and White Rabbit for fiber-optic networks. Nonetheless, GPS is known to suffer from spoofing vulnerabilities [82], and given White Rabbit's relationship to PTP, the impact of asymmetric path attacks presents important questions for ongoing research $[83,84]$. Interestingly, in addition to classical strategies [85], it is possible that QKD can be used to defend against such attacks as well $[86,87]$. As quantum networks evolve, it will be essential to emphasize the security of timing signals to ensure reliable performance.

In general, polarization entanglement seems to be particularly well suited to QLANs, where relatively short and environmentally protected fibers result in low polarization mode dispersion and minimal drift with time. Indeed, with the level of stability obtained in submarine fibers, polarization encoding has been harnessed successfully on scales of approximately $100 \mathrm{~km}$ without active compensation [88]. By replacing the current biphoton source with one covering the entire C-band (1530-1570 nm) or beyond [47,89,90] and leveraging nested WSSs as in Fig. 1(d), one can envision much larger networks utilizing the same technology. As an example, suppose that the bandwidth of the source in Fig. 1(d) is divided into three sections, each frequency correlated. One section is distributed among the outputs of the first WSS and the second section to those of the nested WSS (i.e., the first WSS passes this entire section to the second WSS). This enables each WSS to form a QLAN where nodes in the same QLAN are able to form a fully connected network. The third section can then be routed or divided between the WSSs (on-demand) to form logical links between these two QLANs (internetworking). In this

TABLE III. Accidentals-subtracted link data for both bandwidth allocations.

\begin{tabular}{lccccc}
\hline \hline Allocation & Link & Ch. & Fidelity & $E_{\mathcal{N}}($ ebits $)$ & $R_{E}($ ebits/s) \\
\hline 1 & A-B & 1 & $0.960 \pm 0.005$ & $0.96 \pm 0.01$ & $52.4 \pm 0.6$ \\
& B-C & $2-7$ & $0.925 \pm 0.006$ & $0.95 \pm 0.03$ & $33 \pm 1$ \\
& C-A & 8 & $0.985 \pm 0.001$ & $0.987 \pm 0.005$ & $207 \pm 1$ \\
2 & A-B & 3 & $0.957 \pm 0.004$ & $0.97 \pm 0.02$ & $53.3 \pm 0.8$ \\
& B-C & $1-2$ & $0.959 \pm 0.005$ & $0.98 \pm 0.01$ & $24.8 \pm 0.4$ \\
& C-A & 4 & $0.968 \pm 0.001$ & $0.98 \pm 0.01$ & $320 \pm 4$ \\
\hline \hline
\end{tabular}


way a basic quantum internet, in the literal sense as "interconnected networks," can be realized with small upgrades to the present system.

Because the entanglement paradigm adopted here realizes all-to-all $N$-node connectivity by simultaneously assigning each user $N-1$ frequency channels [42,43,49, 50 ], the histogram for each pair of users sustains an intrinsic amount of background associated with detection events from other channels. Although this could be mitigated by local demultiplexing at each receiver (as emulated in our individual link tests here), the reconfigurability of the flex-grid entanglement paradigm suggests a much more scalable solution: rather than allocating bandwidth for simultaneous all-to-all communications, the WSS could instead establish one-to-one links only, based on user requests [47]. Corresponding to an entanglement graph topology in which each vertex has at most one edge, this approach not only removes the main contribution to crosstalk, but it also leads to a required number of wavelength channels that scales linearly - rather than quadratically — with the number of users. In this way, the flex-grid approach can enjoy the benefits of effective full connectivity (when averaged over all WSS settings) while minimizing bandwidth requirements and noise background. Our current WSS supports 192 channels of 25-GHz width, so that - with a broadband biphoton source spanning the entire C-band-one-to-one connections between nearly 200 nodes could in principle be supported without placing any further demands on the spectral resolution of our devices.

Finally, the question of how best to characterize the generic quality of a quantum network remains open. As we have observed here, optimizing one metric may negatively impact others, and so the best configuration may depend strongly on the application. Interestingly, this goal of quantum network benchmarking mirrors similar objectives in quantum computing, where the concept of quantum volume has been proposed to account for both the number of qubits and gate performance in a consistent framework [91]. So while it remains to be seen what (if any) metric will prove the most informative for evaluating quantum networks, our exploration of an entanglement rate in ebits/s forms an important step in this direction, as it is able to integrate both the quality and quantity of entanglement resources into a single value.

\section{ACKNOWLEDGMENTS}

We thank S. Hicks for assistance in setting up the control plane. This work was performed in part at Oak Ridge National Laboratory, operated by UT-Battelle for the U.S. Department of Energy under contract no. DE-AC0500OR22725. Funding was provided by the U.S. Department of Energy, Office of Science, Office of Advanced Scientific Computing Research, through the Early Career
Research Program and Transparent Optical Quantum Networks for Distributed Science Program (Field Work Proposals ERKJ353 and ERKJ355). N.B.L. acknowledges funding from the Quantum Information Science and Engineering Network (QISE-NET) through the National Science Foundation (1747426-DMR). B.J.L. and Y.Y.P. are sponsored by the U.S. Department of Energy, Office of Science, Basic Energy Sciences, Materials Sciences and Engineering Division (Field Work Proposal ERKCK51). C.E.M. is sponsored by the Intelligence Community Postdoctoral Research Fellowship Program at the Oak Ridge National Laboratory, administered by Oak Ridge Institute for Science and Education through an interagency agreement between the U.S. Department of Energy and the Office of the Director of National Intelligence.

[1] J. I. Cirac, A. K. Ekert, S. F. Huelga, and C. Macchiavello, Distributed quantum computation over noisy channels, Phys. Rev. A 59, 4249 (1999).

[2] J. J. Bollinger, W. M. Itano, D. J. Wineland, and D. J. Heinzen, Optimal frequency measurements with maximally correlated states, Phys. Rev. A 54, R4649 (1996).

[3] V. Giovannetti, Quantum-enhanced measurements: Beating the standard quantum limit, Science 306, 1330 (2004).

[4] V. Giovannetti, S. Lloyd, and L. Maccone, Advances in quantum metrology, Nat. Photon. 5, 222 (2011).

[5] C. H. Bennett and G. Brassard, Quantum cryptography: Public key distribution and coin tossing, Theor. Comput. Sci. 560, 7 (2014).

[6] N. Gisin and R. Thew, Quantum communication, Nat. Photon. 1, 165 (2007).

[7] A. Broadbent, J. Fitzsimons, and E. Kashefi, in 50th Ann. IEEE Sym. Found. Comput. Sci. (IEEE, Atlanta, GA, 2009), p. 517.

[8] S. Barz, E. Kashefi, A. Broadbent, J. F. Fitzsimons, A. Zeilinger, and P. Walther, Demonstration of blind quantum computing, Science 335, 303 (2012).

[9] H. J. Kimble, The quantum internet, Nature 453, 1023 (2008).

[10] S. Wehner, D. Elkouss, and R. Hanson, Quantum internet: A vision for the road ahead, Science 362, eaam9288 (2018).

[11] D. Gottesman, T. Jennewein, and S. Croke, LongerBaseline Telescopes Using Quantum Repeaters, Phys. Rev. Lett. 109, 070503 (2012).

[12] E. T. Khabiboulline, J. Borregaard, K. De Greve, and M. D. Lukin, Optical Interferometry with Quantum Networks, Phys. Rev. Lett. 123, 070504 (2019).

[13] R. Jozsa, D. S. Abrams, J. P. Dowling, and C. P. Williams, Quantum Clock Synchronization Based on Shared Prior Entanglement, Phys. Rev. Lett. 85, 2010 (2000).

[14] P. Kómár, E. M. Kessler, M. Bishof, L. Jiang, A. S. Sørensen, J. Ye, and M. D. Lukin, A quantum network of clocks, Nature Phys. 10, 582 (2014).

[15] P. Kómár, T. Topcu, E. M. Kessler, A. Derevianko, V. Vuletić, J. Ye, and M. D. Lukin, Quantum Network of Atom Clocks: A Possible Implementation with Neutral Atoms, Phys. Rev. Lett. 117, 060506 (2016). 
[16] B. M. Roberts, G. Blewitt, C. Dailey, M. Murphy, M. Pospelov, A. Rollings, J. Sherman, W. Williams, and A. Derevianko, Search for domain wall dark matter with atomic clocks on board global positioning system satellites, Nat. Commun. 8, 1195 (2017).

[17] P. Wcisło et al., New bounds on dark matter coupling from a global network of optical atomic clocks, Sci. Adv. 4, eaau4869 (2018).

[18] V. Scarani, H. Bechmann-Pasquinucci, N. J. Cerf, M. Dušek, N. Lütkenhaus, and M. Peev, The security of practical quantum key distribution, Rev. Mod. Phys. 81, 1301 (2009).

[19] F. Xu, X. Ma, Q. Zhang, H.-K. Lo, and J.-W. Pan, Secure quantum key distribution with realistic devices, Rev. Mod. Phys. 92, 025002 (2020).

[20] D. Gottesman and I. Chuang, Quantum digital signatures, arXiv:quant-ph/0105032 (2001).

[21] P. J. Clarke, R. J. Collins, V. Dunjko, E. Andersson, J. Jeffers, and G. S. Buller, Experimental demonstration of quantum digital signatures using phase-encoded coherent states of light, Nat. Commun. 3, 1174 (2012).

[22] V. Dunjko, P. Wallden, and E. Andersson, Quantum Digital Signatures Without Quantum Memory, Phys. Rev. Lett. 112, 040502 (2014).

[23] M. Hillery, V. Bužek, and A. Berthiaume, Quantum secret sharing, Phys. Rev. A 59, 1829 (1999).

[24] A. Karlsson, M. Koashi, and N. Imoto, Quantum entanglement for secret sharing and secret splitting, Phys. Rev. A 59, 162 (1999).

[25] W. Tittel, H. Zbinden, and N. Gisin, Experimental demonstration of quantum secret sharing, Phys. Rev. A 63, 042301 (2001).

[26] Y.-A. Chen, A.-N. Zhang, Z. Zhao, X.-Q. Zhou, C.-Y. Lu, C.-Z. Peng, T. Yang, and J.-W. Pan, Experimental Quantum Secret Sharing and Third-Man Quantum Cryptography, Phys. Rev. Lett. 95, 200502 (2005).

[27] C. Schmid, P. Trojek, M. Bourennane, C. Kurtsiefer, M. Żukowski, and H. Weinfurter, Experimental Single Qubit Quantum Secret Sharing, Phys. Rev. Lett. 95, 230505 (2005).

[28] W. P. Grice, P. G. Evans, B. Lawrie, M. Legré, P. Lougovski, W. Ray, B. P. Williams, B. Qi, and A. M. Smith, Two-party secret key distribution via a modified quantum secret sharing protocol, Opt. Express 23, 7300 (2015).

[29] B. P. Williams, J. M. Lukens, N. A. Peters, B. Qi, and W. P. Grice, Quantum secret sharing with polarization-entangled photon pairs, Phys. Rev. A 99, 062311 (2019).

[30] H.-J. Briegel, W. Dür, J. I. Cirac, and P. Zoller, Quantum Repeaters: The Role of Imperfect Local Operations in Quantum Communication, Phys. Rev. Lett. 81, 5932 (1998).

[31] C. Elliott, Building the quantum network, New J. Phys. 4, 46 (2002).

[32] M. Peev et al., The SECOQC quantum key distribution network in vienna, New J. Phys. 11, 075001 (2009).

[33] D. Stucki et al., Long-term performance of the swissQuantum quantum key distribution network in a field environment, New J. Phys. 13, 123001 (2011).

[34] M. Sasaki et al., Field test of quantum key distribution in the tokyo qkd network, Opt. Express 19, 10387 (2011).
[35] T.-Y. Chen, J. Wang, H. Liang, W.-Y. Liu, Y. Liu, X. Jiang, Y. Wang, X. Wan, W.-Q. Cai, L. Ju, L.-K. Chen, L.-J. Wang, Y. Gao, K. Chen, C.-Z. Peng, Z.-B. Chen, and J.-W. Pan, Metropolitan all-pass and inter-city quantum communication network, Opt. Express 18, 27217 (2010).

[36] S. Wang et al., Field and long-term demonstration of a wide area quantum key distribution network, Opt. Express 22, 21739 (2014).

[37] Y. Mao, B.-X. Wang, C. Zhao, G. Wang, R. Wang, H. Wang, F. Zhou, J. Nie, Q. Chen, Y. Zhao, Q. Zhang, J. Zhang, T.-Y. Chen, and J.-W. Pan, Integrating quantum key distribution with classical communications in backbone fiber network, Opt. Express 26, 6010 (2018).

[38] J. F. Dynes, A. Wonfor, W. W. S. Tam, A. W. Sharpe, R. Takahashi, M. Lucamarini, A. Plews, Z. L. Yuan, A. R. Dixon, J. Cho, Y. Tanizawa, J. P. Elbers, H. Greißer, I. H. White, R. V. Penty, and A. J. Shields, Cambridge quantum network, npj Quantum Inf. 5, 101 (2019).

[39] P. G. Evans, D. Earl, D. D. Mulkey, R. Newell, G. Peterson, C. Safi, J. L. Tripp, and N. A. Peters, Trusted node QKD at an electrical utility, IEEE Access 9, 105220 (2021).

[40] P. D. Townsend, Quantum cryptography on multiuser optical fibre networks, Nature 385, 47 (1997).

[41] H. C. Lim, A. Yoshizawa, H. Tsuchida, and K. Kikuchi, Wavelength-multiplexed distribution of highly entangled photon-pairs over optical fiber, Opt. Express 16, 22099 (2008).

[42] S. Wengerowsky, S. K. Joshi, F. Steinlechner, H. Hubel, and R. Ursin, An entanglement-based wavelength-multiplexed quantum communication network, Nature 564, 225 (2018).

[43] S. K. Joshi, D. Aktas, S. Wengerowsky, M. Lončarić, S. P. Neumann, B. Liu, T. Scheidl, G. C. Lorenzo, Ž. Samec, L. Kling, A. Qiu, M. Razavi, M. Stipčević, J. G. Rarity, and R. Ursin, A trusted node-free eight-user metropolitan quantum communication network, Sci. Adv. 6, eaba0959 (2020).

[44] P. Toliver, R. Runser, T. Chapuran, J. Jackel, T. Banwell, M. Goodman, R. Hughes, C. Peterson, D. Derkacs, J. Nordholt, L. Mercer, S. McNown, A. Goldman, and J. Blake, Experimental investigation of quantum key distribution through transparent optical switch elements, IEEE Photon. Technol. Lett. 15, 1669 (2003).

[45] N. A. Peters, P. Toliver, T. E. Chapuran, R. J. Runser, S. R. McNown, C. G. Peterson, D. Rosenberg, N. Dallmann, R. J. Hughes, K. P. McCabe, J. E. Nordholt, and K. T. Tyagi, Dense wavelength multiplexing of $1550 \mathrm{~nm}$ QKD with strong classical channels in reconfigurable networking environments, New J. Phys. 11, 045012 (2009).

[46] T. E. Chapuran, P. Toliver, N. A. Peters, J. Jackel, M. S. Goodman, R. J. Runser, S. R. McNown, N. Dallmann, R. J. Hughes, K. P. McCabe, J. E. Nordholt, C. G. Peterson, K. T. Tyagi, L. Mercer, and H. Dardy, Optical networking for quantum key distribution and quantum communications, New J. Phys. 11, 105001 (2009).

[47] I. Herbauts, B. Blauensteiner, A. Poppe, T. Jennewein, and $\mathrm{H}$. Hubel, Demonstration of active routing of entanglement in a multi-user network, Opt Express 21, 29013 (2013).

[48] F. Laudenbach, B. Schrenk, M. Achleitner, N. Vokić, D. Milovančev, and $\mathrm{H}$. Hübel, Flexible cloud/usercentric entanglement and photon pair distribution with 
synthesizable optical router, IEEE J. Sel. Top. Quantum Electron. 26, 6400509 (2020).

[49] N. B. Lingaraju, H.-H. Lu, S. Seshadri, D. E. Leaird, A. M. Weiner, and J. M. Lukens, Adaptive bandwidth management for entanglement distribution in quantum networks, Optica 8, 329 (2021).

[50] F. Appas, F. Baboux, M. I. Amanti, A. Lemaítre, F. Boitier, E. Diamanti, and S. Ducci, Flexible entanglementdistribution network with an AlGaAs chip for secure communications, npj Quantum Inf. 7, 118 (2021).

[51] V. Cerf and R. Kahn, A protocol for packet network intercommunication, IEEE Trans. Commun. 22, 637 (1974).

[52] R. Braden, "Requirements for Internet Hosts - Communication Layers, ” RFC 1122 (1989).

[53] W. K. Wootters and W. H. Zurek, A single quantum cannot be cloned, Nature 299, 802 (1982).

[54] A. Ciurana, V. Martin, J. Martinez-Mateo, B. Schrenk, M. Peev, and A. Poppe, Entanglement distribution in optical networks, IEEE J. Sel. Top. Quantum Electron. 21, 37 (2015).

[55] M. Alshowkan and K. Elleithy, in IEEE Int. Conf. Netw. Arch. Storage (IEEE, Long Beach, CA, 2016), p. 1.

[56] F. Kaiser, A. Issautier, L. A. Ngah, O. Dănilă, H. Herrmann, W. Sohler, A. Martin, and S. Tanzilli, High-quality polarization entanglement state preparation and manipulation in standard telecommunication channels, New J. Phys. 14, 085015 (2012).

[57] J. M. Lukens, K. J. H. Law, A. Jasra, and P. Lougovski, A practical and efficient approach for bayesian quantum state estimation, New J. Phys. 22, 063038 (2020).

[58] IEEE-SA Standards Board, "IEEE Standard for a Precision Clock Synchronization Protocol for Networked Measurement and Control Systems, " IEEE Std 1588-2008 (2008).

[59] M. Lipiński, T. Włostowski, J. Serrano, and P. Alvarez, in IEEE Int. Sym. Precision Clock Sync. Meas. Contr. Commun. (IEEE, Munich, Germany, 2011), p. 25.

[60] R. J. Hughes, T. E. Chapuran, N. Dallmann, P. A. Hiskett, K. P. McCabe, P. M. Montano, J. E. Nordholt, C. G. Peterson, R. J. Runser, R. Sedillo, K. Tyagi, and C. C. Wipf, A quantum key distribution system for optical fiber networks, Proc. SPIE 5893, 589301 (2005).

[61] M. P. Peloso, I. Gerhardt, C. Ho, A. Lamas-Linares, and C. Kurtsiefer, Daylight operation of a free space, entanglement-based quantum key distribution system, New J. Phys. 11, 045007 (2009).

[62] M. Krenn, J. Handsteiner, M. Fink, R. Fickler, and A. Zeilinger, Twisted photon entanglement through turbulent air across vienna, PNAS 112, 14197 (2015).

[63] F. Steinlechner, S. Ecker, M. Fink, B. Liu, J. Bavaresco, M. Huber, T. Scheidl, and R. Ursin, Distribution of highdimensional entanglement via an intra-city free-space link, Nat. Commun. 8, 15971 (2017).

[64] Y. Shi, S. Moe Thar, H. S. Poh, J. A. Grieve, C. Kurtsiefer, and A. Ling, Stable polarization entanglement based quantum key distribution over a deployed metropolitan fiber, Appl. Phys. Lett. 117, 124002 (2020).

[65] G. B. Xavier, G. Vilela de Faria, G. P. Temporão, and J. P. von der Weid, Full polarization control for fiber optical quantum communication systems using polarization encoding, Opt. Express 16, 1867 (2008).
[66] G. B. Xavier, N. Walenta, G. Vilela de Faria, G. P. Temporão, N. Gisin, H. Zbinden, and J. P. von der Weid, Experimental polarization encoded quantum key distribution over optical fibres with real-time continuous birefringence compensation, New J. Phys. 11, 045015 (2009).

[67] S. X. Wang and G. S. Kanter, Robust multiwavelength allfiber source of polarization-entangled photons with builtin analyzer alignment signal, IEEE J. Sel. Top. Quantum Electron. 15, 1733 (2009).

[68] N. Peters, J. Altepeter, E. Jeffrey, D. Branning, and P. Kwiat, Precise creation, characterization, and manipulation of single optical qubits, Quantum Info. Comput. 3, 503 (2003).

[69] A. Poppe, A. Fedrizzi, R. Ursin, H. R. Böhm, T. Lorünser, O. Maurhardt, M. Peev, M. Suda, C. Kurtsiefer, H. Weinfurter, T. Jennewein, and A. Zeilinger, Practical quantum key distribution with polarization entangled photons, Opt. Express 12, 3865 (2004).

[70] C. H. Bennett, D. P. DiVincenzo, J. A. Smolin, and W. K. Wootters, Mixed-state entanglement and quantum error correction, Phys. Rev. A 54, 3824 (1996).

[71] G. Vidal and R. F. Werner, Computable measure of entanglement, Phys. Rev. A 65, 032314 (2002).

[72] M. Horodecki, P. Horodecki, and R. Horodecki, Separability of mixed states: Necessary and sufficient conditions, Phys. Lett. A 223, 1 (1996).

[73] B. J. Pearson and D. P. Jackson, A hands-on introduction to single photons and quantum mechanics for undergraduates, Am. J. Phys. 78, 471 (2010).

[74] P. G. Kwiat, A. J. Berglund, J. B. Altepeter, and A. G. White, Experimental verification of decoherence-free subspaces, Science 290, 498 (2000).

[75] N. A. Peters, J. B. Altepeter, D. Branning, E. R. Jeffrey, T.C. Wei, and P. G. Kwiat, Maximally entangled mixed states: Creation and Concentration, Phys. Rev. Lett. 92, 133601 (2004).

[76] H.-K. Lo, Classical-communication cost in distributed quantum-information processing: A generalization of quantum-communication complexity, Phys. Rev. A 62, 012313 (2000).

[77] A. K. Pati, Minimum classical bit for remote preparation and measurement of a qubit, Phys. Rev. A 63, 014302 (2000).

[78] C. H. Bennett, D. P. DiVincenzo, P. W. Shor, J. A. Smolin, B. M. Terhal, and W. K. Wootters, Remote State Preparation, Phys. Rev. Lett. 87, 077902 (2001).

[79] N. A. Peters, J. T. Barreiro, M. E. Goggin, T.-C. Wei, and P. G. Kwiat, Remote State Preparation: Arbitrary Remote Control of Photon Polarization, Phys. Rev. Lett. 94, 150502 (2005).

[80] J. F. Fitzsimons Private quantum computation: an introduction to blind quantum computing and related protocols, $\mathrm{Npj}$ Quantum Inf. 3, 23 (2017).

[81] H.-H. Lu, E. M. Simmerman, P. Lougovski, A. M. Weiner, and J. M. Lukens, Fully Arbitrary Control of FrequencyBin Qubits, Phys. Rev. Lett. 125, 120503 (2020).

[82] T. E. Humphreys, B. M. Ledvina, M. L. Psiaki, B. W. O'Hanlon, and P. M. Kintner, in Proc. 21st Int. Tech. Mtg. Satell. Div. Inst. Nav. (ION GNSS 2008) (Institute of Navigation (ION), Savannah, GA, 2008), p. 2314. 
[83] R. Annessi, J. Fabini, F. Iglesias, and T. Zseby, Encryption is futile: Delay attacks on high-precision clock synchronization, arXiv:1811.08569 (2018).

[84] J. Lee, L. Shen, A. Ceré, J. Troupe, A. LamasLinares, and C. Kurtsiefer, Asymmetric delay attack on an entanglement-based bidirectional clock synchronization protocol, Appl. Phys. Lett. 115, 141101 (2019).

[85] M. L. Psiaki and T. E. Humphreys, GNSS spoofing and detection, Proc. IEEE 104, 1258 (2016).

[86] J. Bonior, P. Evans, G. Sheets, J. P. Jones, T. Flynn, L. R. O'Neil, W. Hutton, R. Pratt, and T. Carroll, in 2017 IEEE Wireless Commun. Netw. Conf. (WCNC) (IEEE, San Francisco, CA, 2017), p. 1.

[87] H. Dai, Q. Shen, C.-Z. Wang, S.-L. Li, W.-Y. Liu, W.-Q. Cai, S.-K. Liao, J.-G. Ren, J. Yin, Y.-A. Chen, Q. Zhang, F. Xu, C.-Z. Peng, and J.-W. Pan, Towards satellitebased quantum-secure time transfer, Nat. Phys. 16, 848 (2020).
[88] S. Wengerowsky, S. K. Joshi, F. Steinlechner, J. R. Zichi, S. M. Dobrovolskiy, R. van der Molen, J. W. N. Los, V. Zwiller, M. A. M. Versteegh, A. Mura, D. Calonico, M. Inguscio, H. Hübel, L. Bo, T. Scheidl, A. Zeilinger, A. Xuereb, and R. Ursin, Entanglement distribution over a 96-km-long submarine optical fiber, PNAS 116, 6684 (2019).

[89] P. Vergyris, F. Kaiser, E. Gouzien, G. Sauder, T. Lunghi, and S. Tanzilli, Fully guided-wave photon pair source for quantum applications, Quantum Sci. Technol. 2, 024007 (2017).

[90] E. Y. Zhu, C. Corbari, A. Gladyshev, P. G. Kazansky, H.-K. Lo, and L. Qian, Toward a reconfigurable quantum network enabled by a broadband entangled source, J. Opt. Soc. Am. B 36, B1 (2019).

[91] A. W. Cross, L. S. Bishop, S. Sheldon, P. D. Nation, and J. M. Gambetta, Validating quantum computers using randomized model circuits, Phys. Rev. A 100, 032328 (2019). 